\title{
Study on Validation of the OPM Reservoir Simulator by Comparative Solution Project
}

\author{
Fan $\mathrm{H}^{1 *}$, Westford $\mathrm{GD}^{1}$ and $\mathrm{Li} \mathrm{G}^{2}$ \\ ${ }^{1}$ School of Petroleum Engineering, China University of Petroleum, Qingdao, China \\ ${ }^{2}$ Petroleum Exploration \& Production Research Institute, SINOPEC, Beijing, China
}

*Corresponding author: Haijun Fan, School of Petroleum Engineering, China University of Petroleum (East China), Changjiang West 66 Huangdao, East Qingdao, China, Tel: 18561670029; Email: petroengineer@126.com

\section{Research Article}

Volume 4 Issue 6

Received Date: October 21, 2020

Published Date: November 11, 2020

DOI: $10.23880 /$ ppej-16000241

\section{Abstract}

The Open Porous Media (OPM) reservoir simulation toolkit is a free and open-source development in the reservoir simulation world and one that has received very little attention. OPM Flow is a fully-implicit, black-oil simulator capable of running industry-standard simulation models, which encourage open innovation and reproducible research on modeling and simulation of porous media processes. This study validates and assesses the capabilities of OPM Flow comparing with the industry standard ECLIPSE simulator. Several tests were conducted in order to validate the simulator, including a zerobalance test, symmetrical well test, three simulation models based on the SPE Comparative Solution Project, and a real world dataset from the Norne oilfield in Norway. This variety of tests covers a wide range of reservoir types and specific operating conditions which are representative of expected applications of the software. By comparison it is concluded that OPM Flow reservoir simulator can be considered a validated and capable reservoir simulator that is able to compete with Schlumberger ECLIPSE in many cases and shows great potential for future development. In addition, a basic user interface for queuing and running simulations through the OPM Flow simulator was developed using the Python programming language as well as some modifications to the miscible flooding solver.

Keywords: Open Porous Media (OPM); Simulator; Reservoir Simulation; Model Validation

\section{Introduction}

Over the past 50 years, reservoir simulation has evolved from a research area into one of the most flexible and widely used tools in reservoir engineering. Use of reservoir simulation has grown primarily because of its ability to predict the future performance of oil and gas reservoirs over a wide range of operating conditions $[1,2]$. This involves the use of complex software which utilizes reservoir properties in combination with mathematical flow equations to model the fluid behavior of a hydrocarbon reservoir.

Many reservoir simulators have been developed over the years, and currently, Schlumberger ECLIPSE is praised as an industry standard. These simulation software have mostly been closed-source developments and expensive to license. In recent years however, a new software suite has been introduced to the reservoir simulation world, the Open Porous Media Project. This project provides completely free and open-sourced tools for reservoir simulation to the world, a first for the oil industry. The Open Porous Media (OPM) initiative was started in 2009. It was initially founded as collaboration between groups at Equinor (formerly Statoil), SINTEF, the University of Stuttgart, and the University of Bergen, but over time grew larger as several other groups and individuals have joined and contributed. Today the OPM suite of software has mainly been developed by SINTEF, NORCE (formerly IRIS), Equinor, Ceetron Solutions, Poware Software Solutions, and Dr. Blatt HPC-Simulation Software \& Services. The OPM Flow is a reservoir simulator for threephase black-oil problems using a fully-implicit formulation. Specialized variants are also available for problems relating 


\section{Petroleum \& Petrochemical Engineering Journal}

to solvent and polymer use $[3,4]$.

The aim of this study is to validate and assess the capabilities of the OPM Project simulator to see how it compares to the industry standard ECLIPSE simulator. Simulation models based on the SPE Comparative Solution Project will be utilized along with a dataset form the Norne oilfield in Norway to test software in simulation of various reservoir types and specific cases. These same tests will be run on ECLIPSE and the two sets of results will be analyzed. Based on these results, work will be done to improve the OPM tools performance and features.

\section{Methodology}

Reservoir simulations calculate a range of pressures and fluid saturations across a wide area as well as how these change over time as a result of block to block interactions and fluid withdrawals due to production. The validation of reservoir simulation broadly comes from application of the scientific method to determine whether a proposed approach is reliable or not. More specifically, the scientific method requires that we use the most appropriate technology available and that we use data in our computations that are consistent with all measurements available, honoring appropriate ranges of uncertainty.

Black oil simulators are widely used to solve multiphase, multidimensional flow equations for fluids whose properties depend on pressure. Combining flow equations with the continuity equation gives a mass conservation equation for each component $[5,6]$.

Oil:

$$
-\nabla \cdot \frac{\vec{v}_{\boldsymbol{O}}}{\boldsymbol{B}_{\boldsymbol{o}}}-\frac{q_{\boldsymbol{o}}}{\rho_{\text {osc }}}=\frac{\partial}{\partial \boldsymbol{t}}\left(\phi \frac{\boldsymbol{S}_{\boldsymbol{o}}}{\boldsymbol{B}_{\boldsymbol{o}}}\right)(1)
$$

Water:

$$
-\nabla \cdot \frac{\vec{v}_{w}}{B_{w}}-\frac{q_{w}}{\rho_{w s c}}=\frac{\partial}{\partial t}\left(\phi \frac{S_{w}}{B_{w}}\right)
$$

Gas component in oil, water and gas phases:

$$
-\nabla \cdot\left(\frac{\vec{v}_{g}}{\boldsymbol{B}_{g}}+\frac{\boldsymbol{R}_{s o}}{\boldsymbol{B}_{\boldsymbol{o}}} \overrightarrow{\boldsymbol{v}}_{\boldsymbol{o}}+\frac{\boldsymbol{R}_{s w}}{\boldsymbol{B}_{w}} \vec{v}_{w}\right)-\frac{q_{g}}{\rho_{g s c}}=\frac{\partial}{\partial t}\left[\phi\left(\frac{S_{g}}{\boldsymbol{B}_{g}}+\boldsymbol{R}_{s o} \frac{\boldsymbol{S}_{\boldsymbol{o}}}{\boldsymbol{B}_{\boldsymbol{o}}}+\boldsymbol{R}_{s w} \frac{\boldsymbol{S}_{w}}{\boldsymbol{B}_{w}}\right)\right] \text { (3) }
$$

where $\nabla \cdot \vec{v}$ is the divergence of velocity vector and is shorthand for the expression:

$$
\nabla \cdot \overrightarrow{\boldsymbol{v}}=\frac{\partial}{\partial \boldsymbol{x}} \boldsymbol{v}_{\boldsymbol{x}}+\frac{\partial}{\partial \boldsymbol{y}} \boldsymbol{v}_{\boldsymbol{y}}+\frac{\partial}{\partial \boldsymbol{z}} \boldsymbol{v}_{\boldsymbol{z}}
$$

where, $R_{\mathrm{so}}$ is gas solubility in oil; $R_{\mathrm{sw}}$ is gas solubility in water; $B_{\mathrm{o}}$ is oil formation volume factor; $B_{\mathrm{w}}$ is water formation volume factor; $B_{\mathrm{g}}$ is gas formation volume factor; $\mathrm{sc}$-standard conditions (usually $60^{\circ} \mathrm{F}$ and 14.7 psia in oilfield units); $\rho$

is fluid densities; $\phi$ is porosity; $S_{O}, S_{w}$ and $S_{g}$ are phase saturations.

The SPE's Comparative Solution Projects (CSPs) are highly recognized suites of test datasets for specific problems and the hub of conducting independent comparison of reservoir simulation from different dimensions.

Several tests were carried out on the software so as to validate its capabilities. Two of these were simple tests to check the stability of the simulator while another three were be taken straight from the SPE Comparative Solution projects. Finally, the Norne dataset was used to show real world performance as well as for history matching. The Norne dataset is a compilation of real field data from the Norne Oilfield in Norway and represents the greatest test of the reservoir simulators.

It should be noted that this study does not strictly follow these tests as outlined in the SPE comparative studies. While the data and general properties reported may remain the same in a few cases, the exact procedures are not adhered to and tests with multiple cases were reduced to a single case. This was done to keep the study focused and reported results fairly consistent between tests. In order to make a fair comparison between the two software suites, the following measures were taken:

1. The datasets used were identical for both software. There were no changes made unless absolutely necessary in order to make the simulation run. For these cases, the limitations were noted.

2. Both software suites were run on the same computer to better quantify their efficiency and performance.

3. The datasets were run and the results grouped based on the test and software used.

4. In addition to the actual results calculated by the software, the time taken to run the simulation and RAM usage were also recorded.

5. The tool ResInsight was used to plot the summary graphs which will be used for comparing the performance between the software. While ResInsight is an official part of the OPM Project tools, it is simply a way to visualize the data and does not have any special features tied to the output of either simulator, thus providing no advantage to any one simulator.

\section{Results and Discussion}

\section{Zero Balance Test}

The Zero Balance Test results can be seen in Figure 1, which was completed successfully. The model shows no type of fluid flow when all wells are closed off. This attests to the 
stability of both the model and simulator.

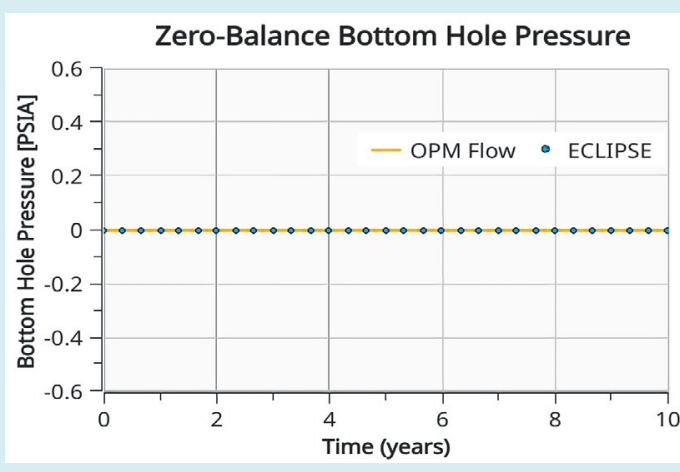

(a) Bottom hole pressure in test 1

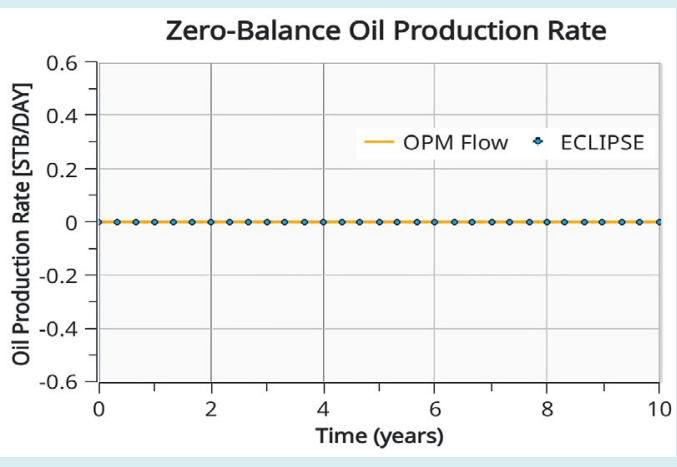

(b) Oil production rate in test 1

Figure 1: Results of Zero-balance Test.

\section{Symmetrical Well Test}

A symmetrical model was constructed and the well positions rotated to 3 separate locations, all exactly sharing the same relevant distance from each other. Figure 2 shows two well orientations used for this study. The wells were rotated in an anticlockwise direction, relative to their starting positions.

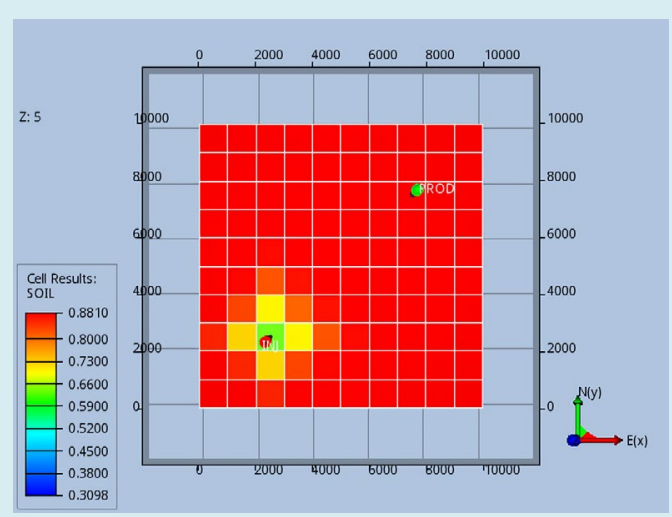

(a) Well location 1

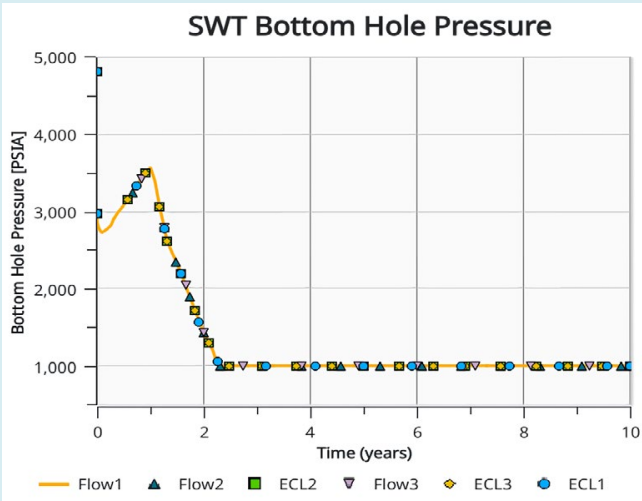

(c) Bottom hole pressures in test 2

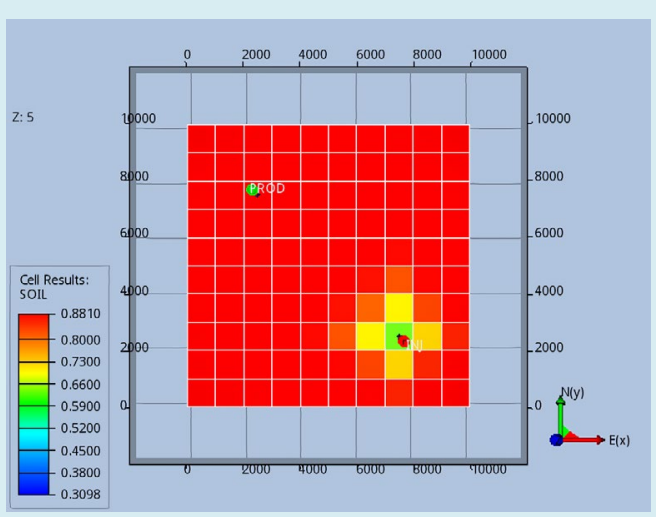

(b) Well location 2

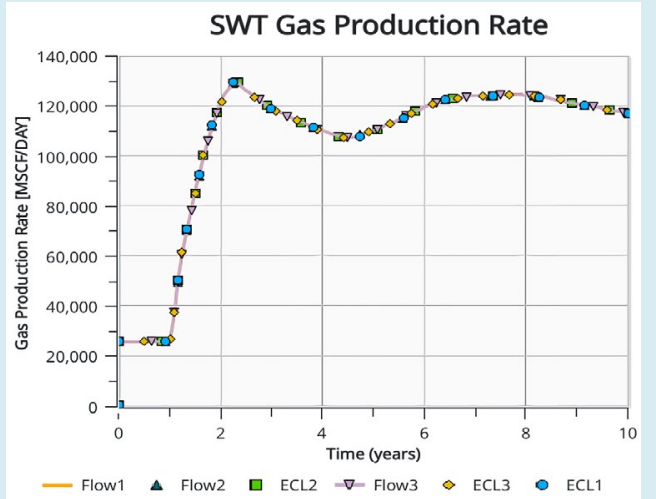

(d) Gas production rates in test 2.

Figure 2: Symmetrical well test orientation and performance. 


\section{Petroleum \& Petrochemical Engineering Journal}

\section{Test 3 SPE 1}

This is the first SPE benchmark. It is a three phase black oil model with nearly immobile water. There is a single producer controlled by bottom-hole pressure, and a single injector injecting gas into the reservoir initially filled with undersaturated oil. Grid dimensions are $10 \times 10 \times 3$. It features
300 blocks total and 3,650 day depletion with gas injection.

Figure 3 shows that the results remained identical between both OPM Flow and Eclipse. There are no perceptible differences present. It can be said that OPM Flow may be considered an equal to Eclipse for the simulation of models such as this one.

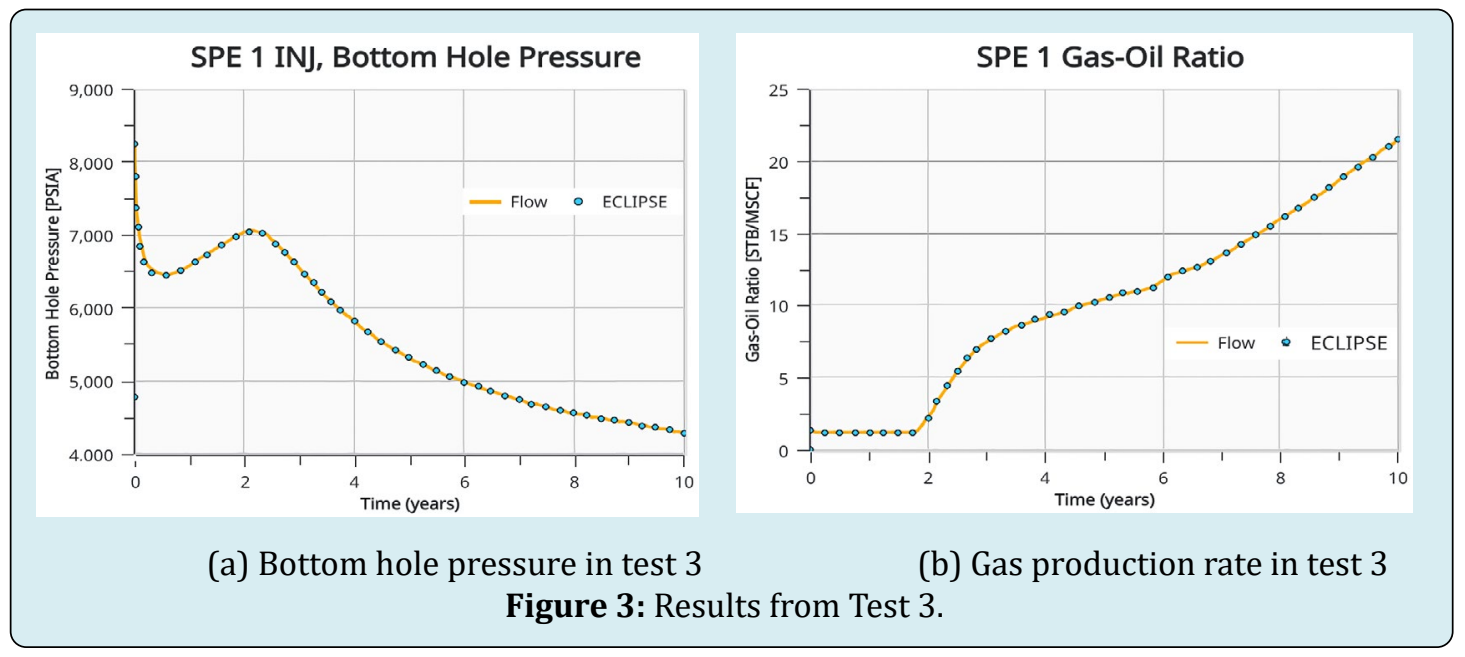

\section{Test 4 SPE 5}

SPE 5 is a six-component volatile oil model [7]. A wateralternating-gas (WAG) injector is located in Cell $(1,1,1)$. A producer located in Cell $(7,7,7)$ and is controlled by the oil production rate. The grid dimensions are $7 \times 7 \times 3$. It features 147 blocks total and goes through a 20 -year WAG injection.
Results between the two simulators are starting to show minor differences. In Figure 4, we can see that starting around the 4 year mark, Eclipse is showing slightly better well performance. This slight variance can also be observed in the total gas production of OPM Flow, which is slightly below that of Eclipse. Despite the difference, it does follow the same pattern however and results are reasonably close.

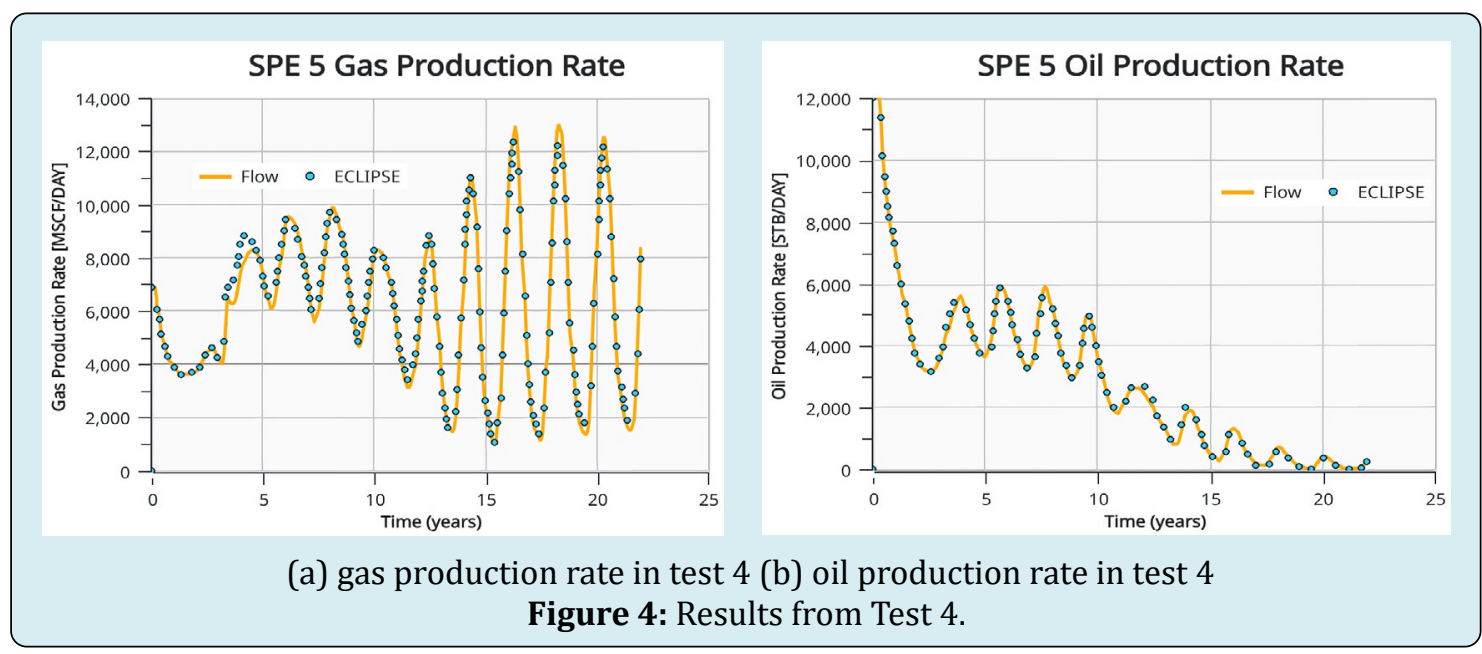

\section{Test 5 SPE 9}

SPE 9 is another, more complex three phase black oil model [8]. It consists of a dipping reservoir produced by a single water injector and 25 producers. This was designed to be a more complex reexamination of black oil simulators.
The grid dimensions are $9 \times 9 \times 6$. It features 486 blocks total with geostatistical descriptions and undergoes a 900 day depletion.

Figures 5 once again show that the results from OPM Flow are practically identical to those from Eclipse. 


\section{Petroleum \& Petrochemical Engineering Journal}

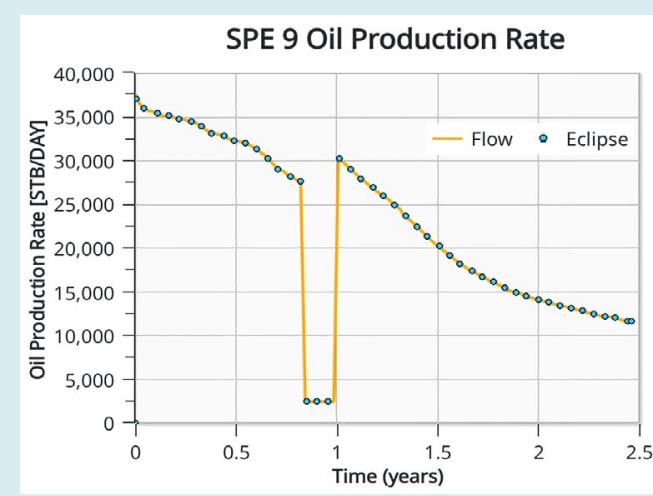

(a) Oil production in test 5 .

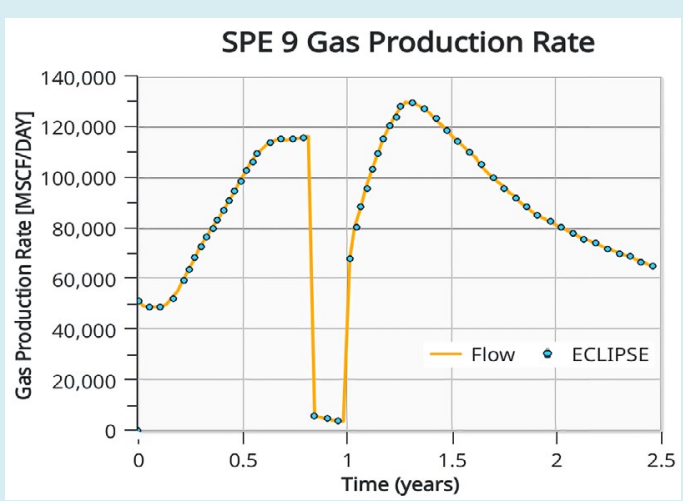

(b) Gas production rate in test 5.

Figure 5: Results from Test 5.

\section{Test 6 Norne Case Study}

The Norne Field is an oil field located in the Norwegian sector of the North Sea. It is located on a horst block in the southern part of the Nordland II. The horst block measures approximately $9 \mathrm{~km} \times 3 \mathrm{~km}$. Before the start of production, free gas was primarily located in the Garn formation and the top of the Ile formation, oil is primarily located in the Ile and Tofte formation, and water is primarily located at the bottom of the Tilje formation [9]. The original oil in place is about 160 million sm ${ }^{3}$. Production began in November 1997, later peaking in 2001 with approximately 11 million $\mathrm{sm}^{3}$ of oil produced. The field has been produced by a total of 22 producers through the end of 2006, and had injected water and gas from nine injectors. By 2012, oil production had decreased to $600,000 \mathrm{sm}^{3}[10,11]$.

The Norne case is a first of its kind comparative case study that uses real data. It mirrors real life reservoir management and would be a helpful tool for benchmarking simulators, as well as evaluating methods of history matching and production optimization.

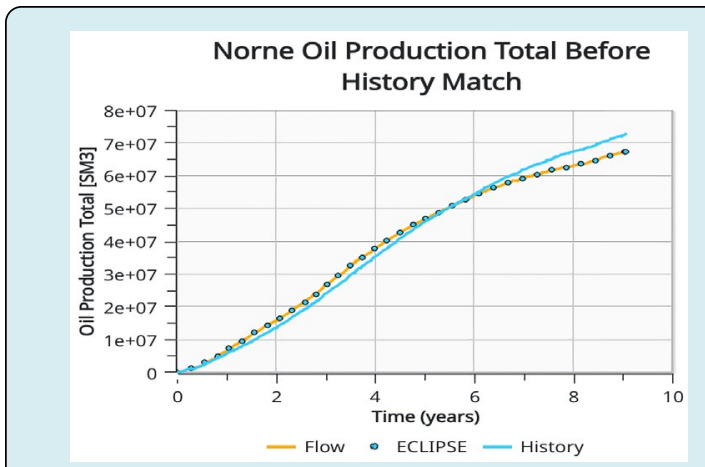

(a) Oil production total before history match.

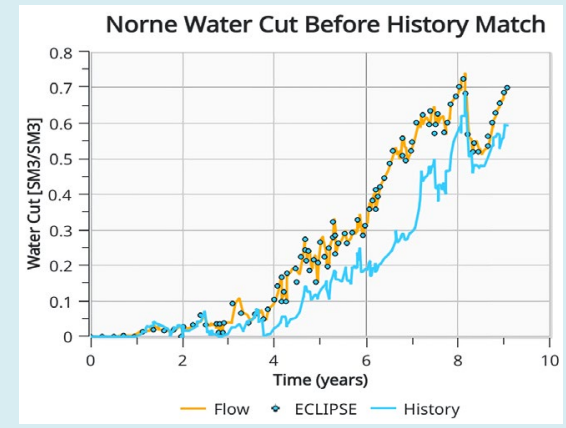

(c) Water cut before history match.

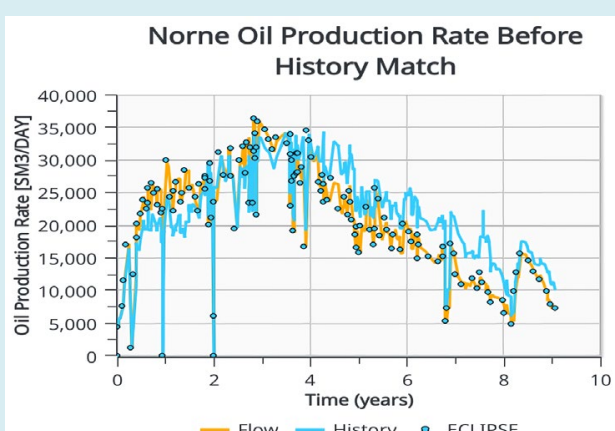

(b) Oil production rate before history match.

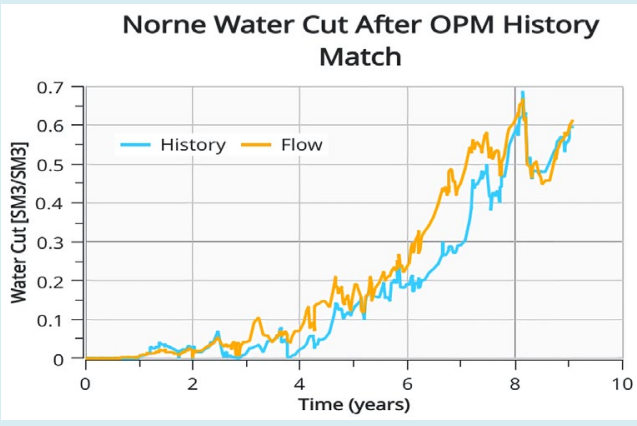

(d) Water cut after OPM history match.

Figure 6: Results from Norne Field. 


\section{Petroleum \& Petrochemical Engineering Journal}

As can be seen in Figures 6, the simulators show near identical results with the Norne dataset. The base dataset however, does not give a good match with the actual production history of the reservoir. This base dataset under performs in every production metric shown. On the following page, the history matched values can be seen. As was stated in the limitations before, values for the Eclipse simulator were omitted due to an issue with oil-water relative permeability.

\section{Performance Tests}

In order to conduct performance tests of the two simulators, the RAM consumption of each run was measured and the average value of the three runs were then calculated. OPM Flow also contains a module which leverages the Open MPI library to run simulations on multiple processing threads, potentially decreasing simulation times. This module was also tested using four CPU threads.

As can be seen in Figure 7, the OPM simulator is quite efficient and often boasts simulation times around 50\% lower than that of Eclipse. The only exception being in the SPE 9 test where Eclipse takes the lead and OPM Flow shows simulation times 50\% longer.

The multi-threaded MPI results for OPM Flow also show significantly lower simulation times over Eclipse with the exception again, being SPE 9. What is odd however is that the MPI results for the first two SPE tests are noticeably higher than those without multi-threading (base OPM Flow). SPE 9 being the outlier with faster simulation time when MPI is activated.

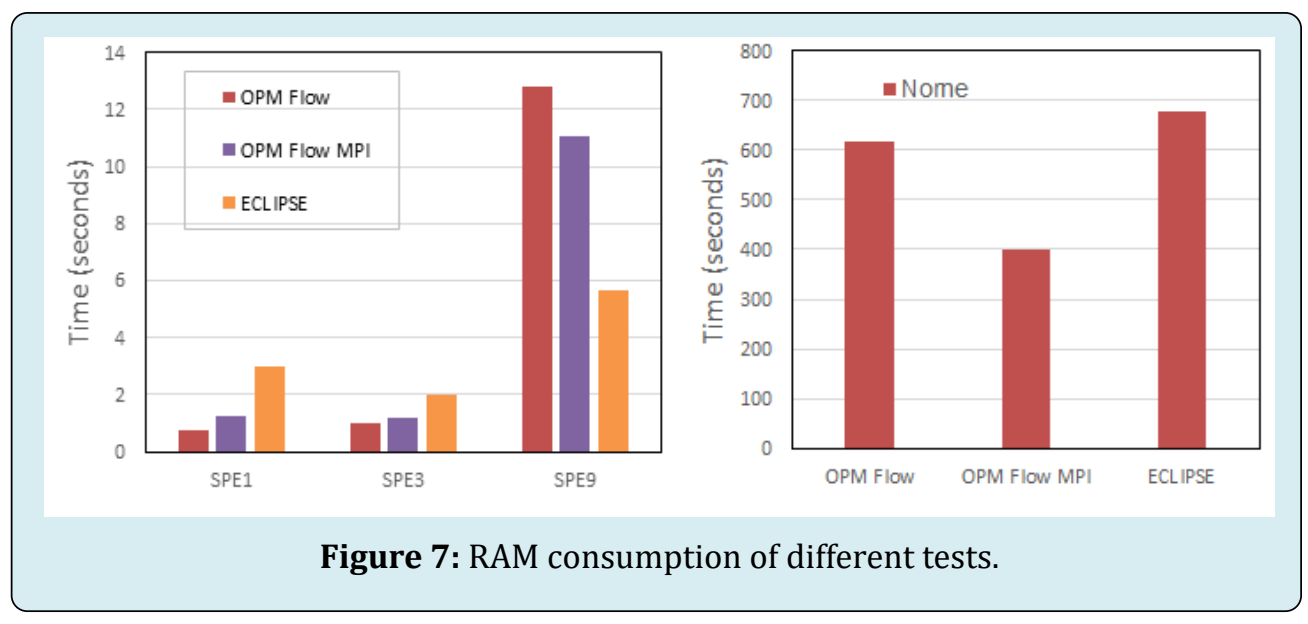

The RAM usage when running the SPE 9 tests was less for OPM Flow than that of Eclipse. However the Norne dataset showed significantly less RAM consumption when run on Eclipse. Using MPI drastically increases RAM consumption on OPM Flow, especially when running the Norne dataset.

\section{Modifications}

\section{Todd-Longstaff Model Modification}

A modification of Todd-Longstaff model for solvent model equations was implemented. The modifications include removing Todd-Longstaff emperical parameter $(\omega)$ and representing the frontal fluid properties explicitly. This modified model separately applies the Todd-Longstaff method in the two different regions of displacement in order to correlate the fluid properties without using the $\omega$ parameter. Applying these changes, the Todd-Longstaff model equations become the following.

When the gas saturation less than critical gas saturation, it is assumed that gas and oil are completely miscible.

$$
\begin{gathered}
\mu_{\boldsymbol{o} e}=\mu_{\boldsymbol{g} \boldsymbol{e}}=\frac{\mu_{\boldsymbol{o}}}{\left(\frac{\boldsymbol{S}_{\boldsymbol{o}}}{\boldsymbol{S}_{\boldsymbol{o}} \boldsymbol{S}_{\boldsymbol{g}}^{\boldsymbol{c}}}\left(\left(\boldsymbol{M}_{\boldsymbol{o}}^{\boldsymbol{c}}\right)^{\frac{1}{4}}-1\right)+1\right)^{4}} \\
\rho_{\boldsymbol{o} \boldsymbol{e}}=\rho_{\boldsymbol{g} \boldsymbol{e}}=\rho_{\boldsymbol{o}}\left(\frac{\boldsymbol{S}_{\boldsymbol{o}}}{\boldsymbol{S}_{\boldsymbol{n}}}\right)_{\boldsymbol{o} \boldsymbol{e}}+\rho_{\boldsymbol{o c}}\left(1-\left(\frac{\boldsymbol{S}_{\boldsymbol{o}}}{\boldsymbol{S}_{\boldsymbol{n}}}\right)_{\boldsymbol{o e}}\right)
\end{gathered}
$$

Where $S_{g}^{C}$ is the critical gas saturation. When the gas saturation great than critical gas saturation, it is assumed that gas and oil are not completely miscible.

The Norne dataset was used to compare the modified Flow simulator and initial Flow simulator. Results are represented by Flow-M for the modified Flow simulator and Flow-I for the Flow simulator in its initial state. Results from the ECLIPSE simulator were also added for reference. 


\section{Petroleum \& Petrochemical Engineering Journal}

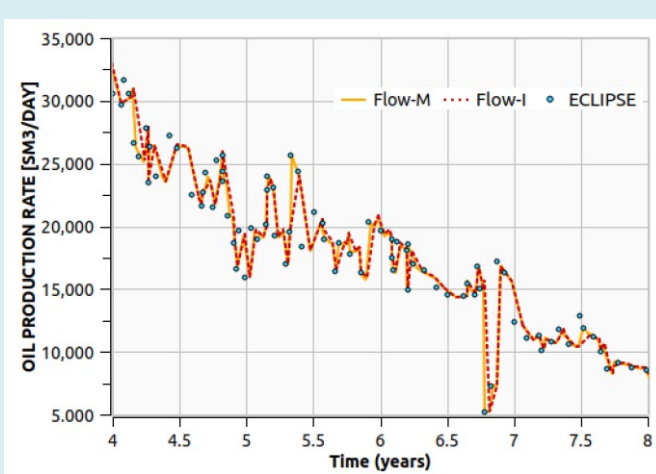

(a) Norne oil production rate

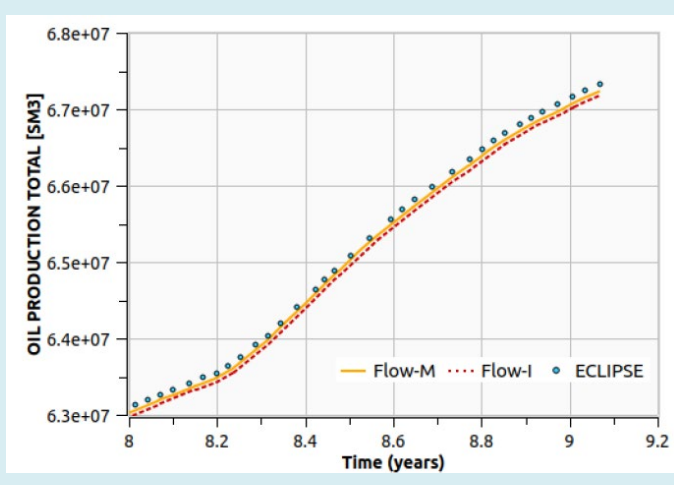

(b) Norne oil production total

Figure 8: Norne Oil Production Change After Model Modification.

Figures 8(a) shows the modified simulator diverging from its unmodified counterpart at multiple different points during oil production. This is most noticeable at some points 4.3, 5.4 and 6.7 on the timescale. Thought these points don't all show an improved production rate, the change seems to be mostly positive. This is reflected in Figure 8(b) where we see that overall oil production is higher. It should be noted however, that the ECLIPSE simulator still shows higher production overall.

From gas and water production profile comparison, we also found that the modified simulator achieves higher peaks in gas production rates than the unmodified simulator and a slightly reduced water production with the modified simulator compared to the unmodified version. While this change does only bring incremental increases to oil and gas production, it is still an improvement over previous results and brings the simulator closer to being on par with the industry standard ECLIPSE simulator.

\section{UI Modifications}

A new application module "OPM Flow Simulation Launcher" has been developed. It features the ability to queue multiple 'DATA' files for consecutive runs through an iteration loop and gives feedback through a real-time terminal output, so the progress of simulations may be monitored. Up to five models can be queued at once and run. The simulation can be run in single or multi-threaded modes through OpenMPI and settings for this are present in the interface.

With the adoption of a graphical user interface, OPM Flow becomes much more user friendly. This encourages the possibility of more people discovering and using this unique simulator.

\section{Conclusions}

The OPM Flow simulator successfully completed tests and showed its ability to handle multiple different reservoir types without issue. These tests validate the OPM Flow simulator as a capable reservoir simulator that can stand toe to toe with the best reservoir simulators in the industry today. In addition, the OPM Flow simulator showcased phenomenal performance when running simulations, often producing lower simulation times compared to Eclipse, especially with the MPI module active [12].

It can be concluded that OPM Flow is a validated and capable reservoir simulator that is able to compete with Schlumberger Eclipse in many cases. While not perfect, it shows great potential for future development. Being free and open source software, it can be easily modified or adapted to suite your needs, and development of extra modules or features is encouraged. As it is still under development, in the coming years, OPM Flow may mature into a very capable tool for the reservoir engineering community.

\section{Acknowledgement}

The author would like to acknowledge the financial support from National Science and Technology Major Project (2016ZX05033005-008).

\section{References}

1. Bouska J, Andy O'Donovan MC, Corbett C, Prange AM, Ryan S (1999) Validating Reservoir Models to Improve Recovery. Oilfield Review pp: 21-35.

2. Branets LV, Ghai SS, Lyons SL, Wu XH (2009) Challenges and Technologies in Reservoir Modeling. Commun Comput Phys 6(1): 1-23.

3. Islam AW, Sepehrnoori K (2013) A Review on SPE's Comparative Solution Projects (CSPs). Journal of Petroleum Science Research 2(4): 167-180. 


\section{Petroleum \& Petrochemical Engineering Journal}

4. Christie MA, Blunt MJ (2001) Tenth SPE Comparative Solution Project: A Comparison of Upscaling Techniques. SPE Reservoir Evaluation \& Engineering 4(4): 10.

5. Fanchi JR (2018) Principles of Applied Reservoir Simulation. $4^{\text {th }}($ Edn.), Gulf Professional Publishing, pp: 364.

6. Odeh AS (1981) Comparison of Solutions to a ThreeDimensional Black-Oil Reservoir Simulation Problem. Journal of Petroleum Technology 33(01): 13-25.

7. Killough JE, Kossack CA (1987) Fifth Comparative Solution Project: Evaluation of Miscible Flood Simulators. SPE Symposium on Reservoir Simulation, Society of Petroleum Engineers, San Antonio, Texas USA, pp: 19.

8. Killough JE (1995) Ninth SPE Comparative Solution Project: A Reexamination of Black-Oil Simulation. Society of Petroleum Engineers, pp: 135-147.
9. Chen Y, Oliver DS (2013) History Matching of the Norne Full Field Model Using An Iterative Ensemble Smoothe. SPE Reservoir Evaluation \& Engineering 17(2): 13.

10. Gjerstad HM, Steffensen I, Skagen JI (1995) The Norne Field Exploration History \& Reservoir Development Strategy. Offshore Technology Conference, Houston, Texas, USA.

11. Kenyon D (1987) Third SPE Comparative Solution Project: Gas Cycling of Retrograde Condensate Reservoirs. Journal of Petroleum Technology 39(08): 981-997.

12. Open Porous Media Initiative Flow [OL].

13. Todd MR, Longstaff WJ (1972) The Development, Testing, and application of a numerical simulator for predicting miscible flood performance. Journal of Petroleum Technology 24(7): 874-882. 